\title{
Intracerebral Transplantation of Stromal Stem Cells: A Hope for Patients Suffering Neurological Discapacity after Hemorrhagic Stroke
}

\author{
Jesús Vaquero, Laura Otero, Celia Bonilla, Concepción Aguayo, \\ Alicia Rodríguez and Mercedes Zurita
}

Neuroscience Research Unit and Rafael del Pino Chair for Neuroscience Research, Hospital Puerta de Hierro-Majadahonda, Joaquín Rodrigo, Madrid, Spain

\begin{abstract}
At present, cell therapy with adult stem cells offers hope for treating neurological diseases considered incurable. In this paper, we review our current experience with delayed intracerebral transplantation of bone marrow stromal cells (BMSC) as a potential treatment of established neurological deficits occurring after intracerebral hemorrhage (ICH). The injection of collagenase IV into basal ganglia of adult Wistar rats causes intracerebral hemorrhage and subsequent neurological deficits that can be quantified by means of functional assessment tests. When the neurological deficit is stabilized, the intracerebral administration of BMSC is followed by a clear functional recovery of the treated animals compared to controls. Furthermore, histological studies show that transplanted stem cells can survive long-term in the injured brain, acquiring a phenotype of neurons and glial cells. These findings suggest that stem cell therapy represents an important field of research, with potential clinical application to treatment of established neurological deficits after brain hemorrhage.
\end{abstract}

Keywords: Bone marrow stromal cells, Cell therapy, Intracerebral hemorrhage, Stroke.

\section{Introduction}

Currently, spontaneous intracerebral hemorrhage (ICH) is a type of stroke that causes significant mortality and morbidity. If the patient survives to initial injury, the bleeding gradually reabsorbed, but generally remain major neurological deficits (Qureshi et al., 2001; Ariensen et al., 2003). In recent years, within the concept of regenerative medicine, the potential of cell therapy to alleviate the functional deficits resulting from acquired brain injuries is gaining in importance. In this line of research, the possibility that intracerebral administration of stem cells achieves neurological recovery in experimental models of brain injury or stroke has been reported (Chen et al., 2001; Bonilla et al., 2009; Otero et al., 2010).
In this paper the authors review their experience with intracerebral bone marrow stromal cells (BMSC) administration to alleviate neurological deficits secondary to an experimental hemorrhagic stroke, and discuss the future clinical perspectives of this therapy.

\section{Experimental Model of ICH}

Adult female Wistar rats were subjected to intracerebral administration of collagenase IV to produce ICH. The animals were fixed to a stereotactic system, and a craniotomy $5 \mathrm{~mm}$ in diameter, on the right parietal bone, at the right of the bregmatic suture, was performed. After exposure of the dura mater, an intracerebral injection of $0.5 \mathrm{IU}$ type IV collagenase (Sigma-Aldrich, Madrid, Spain) dissolved in 2 ? of serum was performed with the aid of a 25 ?l Hamilton

Copyright (C) 2013 Jesús Vaquero, Laura Otero, Celia Bonilla, Concepción Aguayo, Alicia Rodríguez and Mercedes Zurita. This is an open access article distributed under the Creative Commons Attribution License unported 3.0, which permits unrestricted use, distribution, and reproduction in any medium, provided that original work is properly cited. Contact author: Jesús Vaquero E-mail: jvaqueroc@telefonica.net 
syringe attached to a microinjector (mod 310 Stoelting Co., Wood Dale, IL, USA). The injection was made on the bregma reference, with the following coordinates: posterior $0.04 \mathrm{~mm}, 3.5 \mathrm{~mm}$ lateral, ventral $6 \mathrm{~mm}$. This procedure results in an ICH centered in the region of the caudate nucleus (Figure 1).

\section{Bone Marrow Stromal Cells}

It is well known that stem cells are undifferentiated cells that retain the ability to divide throughout life and give rise to cells that can become highly specialized and take the place of cells that die or are lost. In the last years, the use of embryonic stem cells has generated great optimism for therapy of human diseases, but their clinical use remains controversial for ethical and technical reasons, among them the difficulty for their obtaining and the risk of tumor formation. In contrast, adult stem cells have gained importance after the knowledge of the biological properties of bone marrow stromal cells (BMSC). These cells are multipotent adult stem cells located in bone marrow that have proved capable of differentiation, not only into different mesenchymal cells, such as osteoblasts, condrocytes and myocytes, but also into endothelial and neuroectodermal cells (Dezawa et al., 2004; Kotobuki et al., 2004; Zurita et al., 2005; Benayahu et al., 2007; Parr et al., 2007; Zurita et al., 2007). BMSC expresses CD73 and CD105 markers, and also other surface markers which in fact represent molecules of adhesion, such as CD54, CD56, CD90 and CD106. They expand in culture up to sixfold and their biological functions are not altered by ageing.

\section{Preparation and Characterization of Donors BMSC}

In our laboratory, Wistar male rats, weighing between 200-250g were used for obtaining donor BMSC. After sacrificing the animals with a mixture of $70 \% \mathrm{CO}_{2}$ and $30 \% \mathrm{O}_{2}$, the tibias and femurs were isolated and epiphyses cut under sterile conditions. After removing the bone marrow by flushing with a syringe with a No. 26 needle, loaded with alpha-MEM supplemented with antibiotics and 10\% fetal medium, a disintegrated cell suspension was obtained. Bone marrow extracted was placed in complete alphaMEM medium supplemented with antibiotic and $20 \%$ fetal bovine serum. Subsequently, the cells of the bone marrow were disintegrated and then filtered through a nylon mesh of 70 microns. The obtained cell suspension was seeded in a culture flask of $75 \mathrm{~cm}^{2}$ and incubated at $37^{\circ} \mathrm{C}$ with $5 \% \quad \mathrm{CO}_{2}$. After 48 hours incubation, the supernatant containing cell debris and nonadherent cells was removed, leaving adherent cells in culture. Subsequently, the culture was washed with phosphate buffered saline (PBS) pH: 7.4 sterile, subsequently $12 \mathrm{ml}$ of complete alpha-MEM medium with $20 \%$ FBS was added, which was replaced every 48-72 hours, during 14 days. The density of BMSC at culture inception was between $3 \times 10^{3}$ and $5 \times 10^{3}$ cells $/ \mathrm{cm}^{2}$. When the cells reached approximately $90 \%$ confluency, they were subjected to incubation with 3 $\mathrm{ml} 0.25 \%$ trypsin/1 mM EDTA for 4-5 minutes at $37^{\circ} \mathrm{C}$. After this incubation period, trypsin was quenched with $6 \mathrm{ml}$ complete alpha-MEM medium. The obtained cells were centrifuged two times at $1200 \mathrm{rpm}$ for 10 minutes. The pellet finally obtained was diluted in alfa-MEM medium $/ 10 \%$ FBS and was counted using the test of viability of trypan blue. After counting the stem cells, they were cultured again in $75 \mathrm{~cm}^{2}$ flasks, at a concentration of 8000 cells $/ \mathrm{cm}^{2}$ in the presence of $12 \mathrm{cc}$ full complete alfa-MEM medium $/ 10 \%$ FBS. The BMSC used for transplantation were subjected to a third centrifugation at 1200 rpm for 15 minutes. Finally, the pellet was resuspended in saline. Phenotypic characterization of BMSC was performed by flow cytometry, expressing CD29 and CD90 and lacking expression $(\leq 5 \%$ positive) of CD11b, CD45 or CD31.

\section{Cell Therapy with BMSC after ICH}

Given previous studies suggesting the usefulness of local therapy with BMSC after brain and spinal cord injury (Vaquero and Zurita, 2011), in our laboratory we performed a study with adult rats suffering severe functional deficits after the 
provocation of a hemorrhagic stroke. After replacing the animals that died from the injury (this model has a mortality during the first 24 hours of approximately 20\%), we randomly distributed 20 animals suffering ICH in two experimental groups, 10 animals in each: 1) Treated group: The animals of this group were treated with intracerebral injection of $5 \times 10^{6}$ BMSC suspended in $10 \mu$ l of saline, in the area of brain injury, 2 months after ICH. 2) Control group: Consisted of other 10 animals, that were administered the same volume of saline alone, in the same zone, and also 2 months after ICH. The experimental procedures were approved by the Animal Welfare Committee of the Puerta de Hierro-Majadahonda Hospital.

\section{Assessment of Neurological Deficits}

During the follow-up period (2 months from the time of ICH induction, and 6 months after BMSC transplantation), neurologic deficit of our animals was weekly assessed through modified Neurological Severity Score (mNSS) test (Bonilla et al., 2009), and data for each group were recorded as mean \pm standard deviation (SD). Kolmogorov-Smirrow test was performed to study whether the data were normally distributed, and ANOVA test was used for a comparison of the mean scores within each group, at the different time points of follow-up. The statistical analysis was performed by means of the InStat statistical system (v 1.01, GraphPad Software Inc., San Diego, CA), with $\mathrm{p}<0.05$ considered statistically significant.

\section{Histological Studies}

Six months after BMSC administration, the rats were sacrificed for histological studies. For sacrifice, the animals were anesthetized with $8 \%$ sevoflurane in a continuous oxygen flow of $3 \mathrm{l} / \mathrm{min}$, and perfused transcardially with $20 \mathrm{ml}$ heparinized saline followed by $60 \mathrm{ml}$ of $4 \%$ paraformaldehyde in 0.1M PBS ( $\mathrm{pH} 7.4$ ). The brains were then dissected, post-fixed in $4 \%$ formalin for 1-2 days at room temperature, and for each rat, a block containing the zone of the ICH was processed for paraffin sectioning. A series of $5-\mu \mathrm{m}$ thick sections were cut with a microtome through each block and mounted on glass slides for histological observations after hematoxylin-eosin staining.

For identification of endogenous neural stem cells and migrating neuroblasts, immunohistochemical expression of nestin and doublecortin was studied. For it, slides were placed in a boiled citrate buffer $(\mathrm{pH}$ $6)$ in a microwave oven (650-720 w). After rinsing in $\mathrm{PBS}$, the sections were exposed to $3 \% \mathrm{H}_{2} \mathrm{O}_{2}$ for $30 \mathrm{~min}$ to quench endogenous peroxidase activity. Primary antibodies used were directed against nestin monoclonal antibody (1:100; Chemicon International Inc., Temecula, CA, USA) and doublecortin polyclonal antibody (1:300; Santa Cruz Biotechnology, Santa Cruz, CA, USA). The sections were incubated with secondary antibodies conjugated to biotin $(1: 200$; Vector Laboratories Inc., Burlingame, CA, USA). Subsequently, the sections were washed in PBS and incubated with avidin-biotinhorseradish peroxidase complex (Vector Laboratories Inc.). 3',3'-diaminobenzidine (DAB) was used as a chromogen. Control slices, lacking primary or secondary antibodies, were analyzed with each series.

For identification of male donor cells, in situ hybridization studies were performed. For it, $5-\mu \mathrm{m}$ thick sections were dewaxed and rehydrated with xilene and graded ethanol and subsequently digested with proteinase $\mathrm{K}(30 \mu \mathrm{g} / \mathrm{ml})$ for 15 minutes at $37^{\circ} \mathrm{C}$. We used a biotinilated-DNA probe for sequence specificity for murine Sry gene, the sex-determining region of $\mathrm{Y}$ chromosome. Hybridization was performed in a hybridization mixture consisting of $50 \%$ deionized formamide, $2 \%$ salmon test DNA, $10 \%$ dextran sulphate, 10\% 50X Denhardt's solution and $400 \mathrm{ng}$ biotinilated-labeled probe at $50^{\circ} \mathrm{C}$ overnight. For the conventional inmunohistochemistry to visualize the biotinilated-labeled probe, we used a mouse anti-biotin monoclonal antibody (1:100, Jackson ImmunoResearch Laboratories, Inc., Baltimore Pike, USA) and secondary antibodies anti-mouse biotinconjugated antibody $(5 \mu \mathrm{g} / \mathrm{ml}$, Vector 
Laboratories Inc, Burlingame, CA, USA), incubated with avidin-biotin-horseradish peroxidase complex (Vector Inc, CA, USA). DAB was used as a chromogen to visualize under conventional microscopy. The number of grafted cells was determined by counting the positive cells by in situ hybridization in one $5-\mu \mathrm{m}$ slide of each 50 $\mu \mathrm{m}$ corresponding to the lesion zone from each animal.

When double immunohistochemistry stain was performed in order to know a possible neural differentiation of donor cells, the secondary antibody used for identification of male donor cells (Sry-gene-positive) was Rhodamine (TRITC)-conjugated antimouse antibody (1:200, Jackson ImmunoResearch Laboratories, Inc., Baltimore Pike, USA). For identification of neuronal or astrocytic differentiation of donor cells, the primary antibodies used after washing in PBS were anti-NeuN monoclonal antibody (1:500 Chemicon International, Inc. Temecula, CA.) and antiGlial Fibrillary Acidic Protein (PGFA) $(1 \mu \mathrm{l} / \mathrm{ml}, \quad$ Lab Vision Corporation) respectively, using CyTM 2-conjugated anti-mouse antibody (1:200, Jackson ImmunoResearch Laboratories, Inc., Baltimore Pike, USA) as secondary antibody. After washing in PBS, the sections were incubated in Dapi and mounted in Glicerol medium. The slices were visualized by fluorescence microscopy.

\section{Functional and Microscopical Findings after BMSC Transplantation}

The results show a clear effectiveness of this type of cell therapy, achieving neurological recovery in animals with intracerebral transplantation of BMSC compared with controls. The animals receiving saline alone showed slight spontaneous improvement after the induction of ICH, with subsequent stabilization during the follow-up, whereas animals receiving BMSC showed a progressive decrease in neurological deficit during the following months after cell therapy. The difference between groups was considered statistically significant from 2 months after transplantation (Figure 2).

Microscopical findings identified the zone of lesion in all animals as a central cavity near the ventricle, surrounded by a scar wall and filled with different cells. A clear reduction of the cavity created after the hemorrhage could not be observed as result of BMSC administration. Nevertheless, in the animals that received intracerebral BMSC, a great number of immunostained cells showing nestin and doublecortin expression was observed into the lesion zone and in the adjacent subventricular zone (SVZ) compared with the animals that received saline only (Figure 3).

When the vicinity of the lesion zone was studied in the animals that received BMSC, double immunostain identified some cells with Y-chromosome (Sry gene) that showed NeuN or GFAP expression (Figure 4). The mean of cells expressing Sry gene was $17.96 \pm 2.9$ /slide. The percentage of Sry-positive cells co-expressing NeuN was approximately $17 \%$, and the percentage of Sry-positive cells that expressed GFAP was approximately $61 \%$.

\section{Possible Mechanisms of Efficacy}

In recent years, we have obtained evidence suggesting that BMSC transplanted in the injured CNS are able to differentiate into neurons and glial cells, and integrated into the injured parenchyma, which is associated with signs of functional recovery (Zurita and Vaquero, 2004; Zurita and Vaquero, 2006; Zurita et al., 2008; Bonilla et al., 2009; Otero et al., 2010; Otero et al., 2012; Vaquero and Zurita, 2011). Using allogeneic transplantation, we found that intracerebral transplantation of maledonor BMSC into female animals is followed by the identification in the host brain of neurons and astrocytes whose nucleus contains one chromosome $\mathrm{Y}$ (this can be objectified by detection of the Sry gene linked to chromosome $\mathrm{Y}$ by in situ hybridization techniques). It is obvious that the presence of neurons (NeuN expression) and astroglial cells (GFAP expression) with Y-chromosome can only be explained by 
neural differentiation of the male-donor BMSC.

Moreover, it has been reported that BMSC could achieve a beneficial effect, at least in the immediate phases after transplantation, through the release of trophic factors capable of inducing proliferation of neural stem cells in neurogenic regions of the brain (Chen et al., 2005; Vaquero and Zurita, 2011).

On the other hand, it is known that the primary precursors of the adult CNS have been identified in areas called neurogenic niches that appear to be remainings of the embryonic brain, and at least two neurogenic areas have been identified in adult brain: the SVZ and dentate gyrus of the hippocampus. The newly formed neuroblasts migrate from SVZ to the olfactory bulb where they differentiate into interneurons. Tangential migration of neuronal precursor passes through a restricted path forming chains which are surrounded by a mesh consisting of astrocytes processes (Lois and AlvarezBuilla, 1994; Kuhn et al., 1996; Kornack and Rakic, 2001).

Studies in our own laboratory have shown that after ICH, neurogenesis enhanced in the SVZ. The newly formed cells try to replace the cell loss resulting from injury. However, most cells from the neurogenic niche of SVZ suffer cell death by apoptosis during this process and the number of neurons that get integrated into the injured area is not enough to get signs of functional recovery (Otero et al., 2012b). The recent demonstration that cell therapy by intracerebral administration of BMSC significantly increases endogenous neurogenesis (Bonilla et al., 2009; Otero et al., 2012a) provides an explanation for the beneficial effects of this new therapy.

\section{Future Perspectives for Clinical Application in Humans}

To summarize these observations, we conclude that intracerebral administration of BMSC is associated with progressive functional recovery in experimental models of ICH and evidence that at least a certain number of donor-cells survive for a long time in the host brain and can take neuronal and astroglial phenotype. Although it is difficult to understand that the neural differentiation of the transplanted BMSC could lead to a restoration of previously interrupted circuit, the demonstration that BMSC can act as biological pumps releasing neurotrophic factors and increasing endogenous neurogenesis in the brain, provide a reasonable basis for considering the application of these techniques in humans.

Clinical trials could consider the application of cell therapy in acute phase after stroke, especially given the ease with which BMSC could be deposited in the evacuation bed of a cerebral hematoma requiring surgery. However, in this situation it is difficult to assess the impact of cell therapy in the improvement that could show the patient. Furthermore, stromal cells can not be used in acute phase after stroke, because their preparation requires several weeks for expansion in vitro, and therefore the use of BMSC allogeneic should be considered. Although the use of allogeneic BMSC is an option that has proven effective in numerous experimental studies in rodents, there is still no adequate experience on biosafety and efficacy of allogeneic BMSC transplantation in upper mammals or humans. The option of using a mixture of bone marrow mononuclear cells is a possibility to consider as it would allow its use in acute phases alter stroke, but transplantation of a cell population formed only by stromal cells has the advantage of their great specificity to achieve neural transdifferentiation within the host tissue, and ease of expansion, taking Into account the effectiveness of transplantation that

appears to be dose-dependent (Vaquero and Zurita, 2009; Vaquero and Zurita, 2011). In these circumstances, it seems logical to raise the first clinical trials in humans as an attempt to alleviate chronic neurological deficits after hemorrhagic stroke, and the use of autologous BMSC. On the other hand, the experience gained in our own laboratory on traumatic brain 
injury models suggest that the degree of severity of injury is an important factor in achieving therapeutic efficacy (Bonilla et al., 2012); a finding that should be taken into account in these trials.

A critical factor that must also be considered is the medium of cell suspension, which largely determines survival and neural differentiation of BMSC (Zurita et al., 2010). Finally, although the local administration seems the most appropriate route in later stages after brain injury (Vaquero et al., 2006; Bonilla et al., 2009), it still do not have enough experimental evidence about the potential for subarachnoid administration and although it is difficult for this route to arrive to a large number of cells at the injury site, it obviously can be a complementary management of local administration (Vaquero and Zurita, 2009; Vaquero and Zurita, 2011).

\section{Conclusions}

In recent years, there is growing interest about the therapeutic potential of adult stem cells. Among them, the BMSC seems set to a cell type capable of reducing neurological deficits after acquired cerebral lesions. Taking into account the experience gained in preclinical animal models, autologous BMSC transplantation can now be proposed as a therapeutic strategy and a possible hope for many patients suffering hemorrhagic stroke.

\section{References}

Ariesen, M. J., Claus, S. P., Rinkel, G. J. A. \& Algra, A. (2003). "Risk Factors for Intracerebral Hemorrhage in the General Population: A Systematic Review," Stroke 34, 2060-2065.

Benayahu, D., Akavia, U. D. \& Shur, I. (2007). "Differentiation of Bone Marrow Stroma-Derived Mesenchymal Cells," Current Medical Chemistry 14, 173-179.

Bonilla, C., Zurita, M., Otero, L., Aguayo, C., Rico, M. A. \& Vaquero, J. (2012). "The Severity of Brain Damage Determines Bone Marrow Stromal Cell Therapy Efficacy in a
Traumatic Brain Injury Model," Journal of Trauma-Injury Infection and Critical Care, 72, 1203-1212.

Bonilla, C., Zurita, M., Otero, L., Aguayo, C. \& Vaquero, J. (2009). "Delayed Intralesional Transplantation of Bone Marrow Stromal Cells Increases Endogenous Neurogenesis and Promotes Functional Recovery after Severe Traumatic Brain Injury," Brain Injury 23, 760-769.

Chen, J., Li, Y., Wang, L., Zhang, Z., Lu, D., Lu, M. \& Chopp, M. (2001). "Therapeutic Benefit of Intravenous Administration of Bone Marrow Stromal Cells after Cerebral Ischemia in Rats," Stroke 32, 1005-1011.

Chen, Q., Long, Y., Yuan, X., Zou, L., Sun, J., Chen, S., Perez-Polo, J. R. \& Yang, K. (2005). "Protective Effects of Bone Marrow Stromal Cell Transplantation in Injured Rodent Brain: Synthesis of Neurotrophic Factors," Journal of Neuroscience Research, 80, 611619.

Dezawa, M., Kanno, H., Hoshino, M., Cho, H., Matsumoto, N., Itokazu, Y., Tajima, N., Yamada, H., Sawada, H., Ishikawa, H., Mimura, T., Kitada, M., Suzuki, Y. \& Ide, C. (2004). "Specific Induction of Neuronal Cells from Bone Marrow Stromal Cells and Application for Autologous Transplantation," Journal of Clinical Investigation 113, 1701-1710.

Kornack, D. R. \& Rakic, P. (2001). "The Generation, Migration, and Differentiation of Olfactory Neurons in the Adult Primate Brain," Proceedings of the National Academy of Sciences of the United Stetes of America 98, 4752- 4757.

Kotobuki, N., Hirose, M., Takakura, Y. \& Ohgushi, H. (2004). "Cultured Autologous Human Cells for Hard Tissue Regeneration: Preparation and Characterization of Mesenchymal Stem Cells from Bone Marrow," Artificial Organs 28, 33-39.

Kuhn, H. G., Dickinson-Anson, H. \& Gage, F. H. (1996). "Neurogenesis in the Dentate Gyrus of the Adult Rat: Age-Related Decrease of Neuronal Progenitor Proliferation," Journal of Neuroscience 16: 2027-2033. 
Lois, C., Alvarez-Buylla, A. (1994). "Long Distance Neuronal Migration in the Adult Mammalian Brain," Science 5162, 11451148.

Otero, L., Bonilla, C., Aguayo, C., Zurita, M. \& Vaquero, J. (2010). 'Intralesional Administration of Allogenic Bone Marrow Stromal Cells Reduces Functional Deficits after Intracerebral Hemorrhage,' Histology and Histopathology, 25, 453-461.

Otero, L., Zurita, M., Bonilla, C., Aguayo, C., Rico, M. A., Rodríguez, A. \& Vaquero, J. (2012a). "Allogeneic Bone Marrow Stromal Cell Transplantation after Cerebral Hemorrhage Achieves Cell Transdifferentiation and Modulates Endogenous Neurogenesis," Cytotherapy 14, 34-44.

Otero, L., Zurita, M., Bonilla, C., Rico, M. A., Aguayo, C., Rodríguez, A. \& Vaquero, J. (2012b). "Endogenous Neurogenesis after Intracerebral Hemorrhage," Histology and Histopathology 27, 303-315.

Parr, A. M., Tator, C. H. \& Keating, A. (2007). "Bone Marrow-Derived Mesenchymal Stromal Cells for the Repair of Central Nervous System Injury," Bone Marrow Transplantation 40, 609-619.

Qureshi, A. I., Tuhrim, S., Broderick, J. P., Batjer, H. H., Hondo, H. \& Hanley, D. F. (2001). "Spontaneous Intracerebral Hemorrhage," New England Journal of Medicine, 344: 1450-1460.

Vaquero, J. \& Zurita, M. (2009). “Bone Marrow Stromal Cells for Spinal Cord Repair: A Challenge for Contemporary Neurobiology," Histology and Histopathology 24, 107-116.

Vaquero, J. \& Zurita, M. (2011). "Functional Recovery after Severe CNS Trauma: Current Perspectives for Cell Therapy with Bone Marrow Stromal Cells," Progress in Neurobiology 93, 341-349.

Vaquero, J., Zurita, M., Oya, S. \& Santos, M. (2006). "Cell Therapy Using Bone Marrow Stromal Cells in Chronic Paraplegic Rats: Systemic or Local Administration?," Neuroscience Letters 398, 129-134.
Zurita, M., Otero, L., Aguayo, C., Bonilla, C., Ferreira, E., Parajón, A. \& Vaquero, J. (2010). "Cell Therapy for Spinal Cord Repair: Optimization of Biologic Scaffolds for Survival and Neural Differentiation of Human Bone Marrow Stromal Cells," Cytotherapy 12, 522-537.

Zurita, M. \& Vaquero, J. (2004). "Functional Recovery in Chronic Paraplegia after Bone Marrow Stromal Cells Transplantation," Neuroreport 15, 1105-1108.

Zurita, M. \& Vaquero, J. (2006). "Bone Marrow Stromal Cells can Achieve Cure Ofchronic Paraplegic Rats: Functional and Morphological Outcome One Year after Transplantation," Neurosciences Letters 402, 51-56.

Zurita, M., Vaquero, J., Bonilla, C., Santos, M., De Haro, J., Oya, S. \& Aguayo, C. (2008). "Functional Recovery of Chronic Paraplegic Pigs after Autologous Transplantation of Bone Marrow Stromal Cells," Transplantation 86, 845-853.

Zurita, M., Vaquero, J., Oya, S., Bonilla, C. \& Aguayo, C. (2007). "Neurotrophic SchwannCell Factors Induce Neural Differentiation of Bone Marrow Stromal Cells," Neuroreport 18, 1713-1717.

Zurita, M., Vaquero, J., Oya, S. \& Miguel, M. (2005). "Schwann Cells Induce Neuronal Differentiation of Bone Marrow Stromal Cells," Neuroreport 16, 505-508. 